\title{
Vehicle Scheduling Optimization Based on Chaos Ant Colony Algorithm in Emergency Rescue
}

\author{
Lingxia Liu ${ }^{1}$ Xuexia $\mathrm{Gao}^{2}$ and Qiang Song ${ }^{3}$ \\ ${ }^{1}$ School of Software Engineering, Anyang Normal University, Anyang, 455000, China \\ ${ }^{2}$ Computer School, Xinxiang University, Xinxiang, 453000, China \\ ${ }^{3}$ Computer School, Anyang Institute of Technology, Anyang, 455000, China \\ aysq168@163.com,llx@aynu.edu.cn
}

\begin{abstract}
Aiming to the demand and characteristics of vehicle scheduling optimization problem in emergency rescue, this paper establishes a multi-objective optimization model, which takes minimizing variable bidirectional distance, path risk and cost as the optimization target. To avoid the prematurely falling into local optimization of ant colony system(ACS)algorithm, and to improve the algorithm adaptability, computational efficiency and the quality of optimal solution, this paper proposed and realized a chaos-based improved ant colony system algorithm, which can overall updates the chaos disturbance to pheromone. Simulation results show that the algorithm is feasible, which can well meet the demand of vehicle scheduling optimization in emergency rescue.
\end{abstract}

Keywords: Emergency, vehicle scheduling, chaos, ant colony system

\section{Introduction}

In recent years, natural disasters such as earthquake and flood seriously threaten human life and property safety. When natural disasters happen, usually a large number of emergency supplies are needed. Scientific and reasonable allocation of resources can play the effect of half the work with double results for emergency rescue. Chunlin Liu etc., [1] studied the optimization model of emergency supplies scheduling; Zhengwen He etc., [2] studied the emergency supplies scheduling of vehicle routing problem based on time window; Ziyao Li [3] studied vehicle routing problem with a single starting point and end point in the multiobjective emergency rescue; Wuyangliu [4] built a logistics optimization and scheduling model in the emergency disposal, ant colony algorithm was used for solving problem. Aiming to the characteristics of emergency logistics, Zhiyu $\mathrm{Xu}$ etc., [5] established the partial distribution vehicle routing planning model, using the max-min ant colony algorithm to solve problem; Liansheng Tang etc., [6] proposed a kind of ant colony clustering optimized algorithm to solve multi-objective optimization problem under the emergency logistics distribution; Minghua Chen etc., [7] created the general emergency logistics vehicle scheduling model with non-full loads, and the artificial immune algorithm is used for solving.

Because emergency rescue vehicle scheduling is interfered by multi-objective and all kinds of uncertain information, making the construction and solving of such a problem become more complex. From the existing literatures, the established models in most literatures still follow research perspectives of business issues; the excavation is not deep enough to the particularity of emergency rescue. Therefore, based on the analysis of emergency rescue vehicle scheduling decision environment and the key factors, this paper makes necessary 
improvements to the determination of emergency rescue multi-objectives, modeling method and algorithm, in order to more accord with the actual situation of emergency rescue.

\section{The Description and Basic Assumption of Emergency Rescue Vehicle Scheduling Problem}

The scheduling optimization problem of emergency rescue vehicle [8] can be briefly described as follows: in the emergency network with a supply and demand relationship, there is a distribution center of emergency rescue, number of vehicles, and several rescue points, the vehicle routes are required to be reasonable arranged, under the emergency environment and conditions limitation, the emergency supplies are transported from the distribution center to each rescue site, making the objective function to achieve the overall optimization.

In order to emphasis the key points, the scheduling problem of emergency rescue vehicle is made appointment according to the following basic agreement:

a) Emergency rescue center has enough transport vehicles to implement rescue, the number of rescue points do not exceed the capacity of a vehicle (or team) in one line, and the sum of each line is not more than the sum of on-board limitation;

b) Each rescue point only has one vehicle (or team) responsible for distribution, each vehicle starts from the distribution center of emergency rescue, and immediately returns back to distribution center after completing the distribution task;

c) In the rescue activities, all rescue points should be traversed;

d) The position of demand points and the quantity of vehicles are determined;

e) Only consider the emergency material distribution, temporarily do not consider personnel situation.

\section{The Multi-Objective Determination of Emergency Rescue and Data Evaluation}

The vehicle scheduling problem of emergency rescue implements the emergency events, which highlights the time urgency. On the processing of time, a lot of literatures adopt the method of the general vehicle scheduling model, which is using time window to solve. In fact, for emergency rescue, this setting is not appropriate, because the time for rescue object services is not the key of the problem; the actual transit time of path under uncertain environment is the main factor. In emergency network, the traveling distance is often uncertain, particularly after the natural disasters such as earthquake and floods, the road will be interrupted or destroyed, which makes the conditions of going and returning different. Therefore, from the perspective of real-time dynamic decision-making, the variable two-way distance ought to be considered in the application model.

At the same time, while a serious disaster occurs, the emergency rescue itself is often a very dangerous activity, if the emergency vehicle dispatching is not appropriate, not only the expected rescue effect cannot be achieved, but also which may cause the new possible losses of life and property. Therefore, in the emergency rescue vehicle scheduling problem the safety of the rescue should be considered.

Although the emergency rescue has weak economy characteristics, the cost also cannot be ignored. For this reason, this article selects the risk, variable two-way distance and cost as the object to build multi-objective optimization model. In the uncertain environments, the expert evaluation method is a relatively simple method of data acquisition. This method can make full use of the expert's knowledge, experience and judgment to obtain decision data quickly, when there are great changes, which also favors the revaluation. 
Unlike straight line distance and static distance, at first an expert should be asked to give evaluation value for the two-way distance of each route section. If emergency rescue center is marked with 0 , there are $n$ rescue points, the distance matrix can be written as follows:

$$
D=\left(d_{i j}\right)_{(n+1) \times(n+1)} \quad i, j=0,1, \ldots, n
$$

In the expression: matrix element $d$ represents the distance evaluation value from the rescue point $\mathrm{i}$ to $\mathrm{j}$ of this route sections. In the evaluation, if two points can set up multiple traffic channel (e.g., road, sea and air channel, etc.), which may be the current priority of the channels; if the road is completely blocked or damaged, there will be a big number which represents the road is difficult to pass.

In the same method, the cost matrix $\mathrm{C}$ is given. Here in addition to normal fuel costs, the cost might include the spending of road tolls, such as temporarily repairing a road, bridge and other expenses.

$$
C=\left(C_{i j}\right)_{(n+1) \times(n+1)} \quad i, j=0,1, \ldots, n
$$

Road safety can directly ask decision makers to make value judgment within the scope of $\{1,2,3,4,5\}$, the value is bigger, it is safer, such as level 5 represents the safest, level 4 is the second, the most unsafe is 1 . The safety factor evaluation matrix is

$$
S=\left(S_{i j}\right)_{(n+1)(n+1)} \quad i, j=0,1, \ldots, n
$$

\section{Mathematical Model}

Assumes that the emergency rescue distribution center $(i, j=0)$ has $K(k=1, \ldots, K)$ vehicles, there are $N(i, j=1, \ldots, N)$ rescue sites, the capacity of each vehicle is $Q$.The variables and parameters are defined as follows:

$$
\begin{aligned}
& x_{i j}^{k}= \begin{cases}1 & \text { vehicle } k \text { passes rescue site } i \text { and } j \\
0 & \text { others }\end{cases} \\
& y_{i k}= \begin{cases}1 & \text { vehicle } k \text { provide distribution service for rescue site } i \\
0 & \text { others }\end{cases}
\end{aligned}
$$

$g_{i}$ is the distribution number that the rescue point $\mathrm{i}$ needs; ${ }^{u_{i}}$ is the distribution service order for rescue point $i$, then multiple-objective function can be determined as follows:

$$
\begin{aligned}
\min D & =\sum_{i=0}^{N} \sum_{j=0}^{N} \sum_{k=1}^{K} d_{i j} x_{i j}^{k} \\
\min C & =\sum_{i=0}^{N} \sum_{j=0}^{N} \sum_{k=1}^{K} c_{i j} x_{i j}^{k} \\
\max S & =\sum_{i=0}^{N} \sum_{j=0}^{N} \sum_{k=1}^{K} s_{i j} x_{i j}^{k}
\end{aligned}
$$

In above expressions, the optimization goal of the problem has three issues, respectively which are the shortest traffic distance, the minimum cost and the largest safety coefficient.

The constraints are as follows: 


$$
\begin{aligned}
& \sum_{i=0}^{N} g_{i} y_{i k} \leq Q \quad k=1,2, \ldots, k \\
& \sum_{k=1}^{K} y_{i k}=\left\{\begin{array}{lr}
1 & i=1,2, \ldots, N \\
K & i=0
\end{array}\right. \\
& \sum_{i=0}^{N} x_{i j}^{k}=y_{j k} \quad j=0,1,2, \ldots, N ; \quad K=1,2, \ldots, K \\
& \sum_{j=0}^{N} x_{i j}^{k}=y_{i k} \quad i=0,1,2, \ldots, N ; \quad K=1,2, \ldots, K \\
& x_{i j}^{k}=0 \quad \forall i=j \quad k=1,2, \ldots, K \\
& u_{i}-u_{j}+(N+1) \sum_{k} x_{i j}^{k} \leq N \\
& i \neq j, \quad i=1,2, \ldots, N ; \quad j=1,2, \ldots, N \\
& x_{i j}^{k} \in\{0,1\}, \quad y_{i k} \in\{0,1\}, \quad u_{i}>0
\end{aligned}
$$

Constraints (4) represents vehicle distribution amount does not exceed the limit of vehicle load; Constraints (5) represents each rescue point has only one vehicle being responsible for the distribution, and there are $\mathrm{K}$ vehicles starting from distribution center; Constraints (6) represents the vehicle A only travels into the rescue point that assigns to its transport task, and only runs out of the rescue point that assigned to its transport task; Expression (7) represents the constraints that is used to prevent a vehicle to produce multiple loops.

\section{The Design of Chaos Disturbance to Improve Ant Colony System Algorithm}

Vehicle scheduling algorithm can be divided into two categories. The first category is based on the research of mathematical programming or network analysis method, the main representative is to use mathematical programming or maximum, minimum flow algorithms of network. This kind of method can effectively solve the problems of the vehicle and more accurate, but which is not suitable for large-scale problem solving, there exists the combination explosion phenomenon. The second category is based on the research of artificial intelligence algorithms. Since the vehicle scheduling problem is clear to be a NPcomplete problem, most of the research focuses on vehicle scheduling are transferred to using a variety of intelligent heuristic algorithm to solve the vehicle scheduling problem. Ant colony algorithm is a kind of swarm intelligence algorithm, which is widely used in recent years. But when the population size is larger, ant colony algorithm is prone to premature, stagnation phenomenon [9, 10], which hinders its further application.

Chaos status widely exists in natural and social phenomenon, with the unique properties of regularity, randomness, ergodicity. Chaotic motion can traverse all the status without repeat within a certain range according to its "law". Therefore, the characteristics of chaotic variables can be fully applied to optimize searching mechanism of ant colony algorithm, and keep the diversity of solution space, so as to improve the algorithm optimal performance [11, 12] of global searching.

To make the algorithm of vehicle scheduling model more efficient and reliable under emergency rescue, in the greatest degree for emergency rescue to save time, this article is 
based on more excellent ant colony system algorithm $[13,14]$ in ant colony algorithm, combing with the chaos disturbance mechanism to design the algorithm in this model.

\subsection{The Settings of State Transition Probability}

The ants number of ant colony is set to $\mathrm{m}$, the heuristic degree of road section from rescue point $i$ to $j$ is $\eta_{i j}$, if $\eta_{i j}=1 / d_{i j}, d_{i j}$ represents the distance evaluation value of road section from rescue point $\mathrm{i}$ to $\mathrm{j}$, at the same time the heuristic information ${ }^{C_{i j}}$ and $S_{i j}$ are introduced, which respectively represent the cost assessment and safety assessment values of the road section from the rescue point $\mathrm{i}$ to $\mathrm{j},{ }^{\tau_{i j}}$ is used to represent the pheromone strength from rescue point $\mathrm{i}$ to $\mathrm{j}$. If $\mathrm{j}$ is set as the next rescue point of ant, the transition probability formula of ant $\mathrm{k}$ from the rescue point $\mathrm{i}$ to $\mathrm{j}$ can be confirmed according to expression (8).

$$
P_{i j}^{k}(t)=\left\{\begin{array}{cl}
\frac{\tau_{i j}(t)^{\alpha} \eta_{i j}^{\beta} c_{i j}^{-\gamma} s_{i j}^{\delta}}{\sum_{j \in \text { allowed }_{k}(t)} \tau_{i j}(t)^{\alpha} \eta_{i j}^{\beta} c_{i j}^{-\gamma} s_{i j}^{\delta}} & \text { if } \mathrm{j} \in \text { allowed }_{k}(t) \\
0 & \text { others }
\end{array}\right.
$$

In the expression (8), allowed $_{k}(t)=(1,2, \ldots, n)-t a b u_{k}$ represents the next point that ant $k$ is allowed to select. Tabu table $\operatorname{tabu}_{k}(1,2, \ldots, m)$ is used to record the points that ant $k$ has passed at $t$ moment, which does not allow ants to repeat in this cycle, after the cycle completes the tabu list will be empty.

$\alpha$ reflects the importance of the accumulated pheromone of ants in the movement process, $\beta, \gamma$, and $\delta$ reflect the relative importance of each heuristic information while the ant chooses path, their value can reflect the preference degree of timeliness, economy and safety by the emergency decision maker, which can be conformed according to the rescue specific environment and delivery task. If the preference degree is very high, the corresponding parameter selects the larger number; otherwise, less value is desirable, and even it can be set to zero.

To prevent algorithm to be prematurely trapped in local optimal solution, it can be improved to use the following method to choose the next visited point, in order to improve the diversity of route choice.

$$
j=\left\{\begin{array}{cl}
\arg \left\{\max _{j=\text { allowed }_{k}(t)}\left[\tau_{i j}(t)^{\alpha} \eta_{i j}^{\beta} c_{i j}^{-\gamma} s_{i j}^{\delta}\right]\right\} & \text { while } q \leq q_{0} \\
J & \text { others }
\end{array}\right.
$$

$q_{0} \in(0,1)$ is a constant, $\mathrm{q}$ is the random number between $0 \sim 1$. When $q>q_{0}, \mathrm{~J}$ is calculated according to expression (8).

Vehicle scheduling optimization needs to make a route choice, at the same time to consider the load limit of each vehicle, if the above choice makes the vehicle load rate is greater than 1 , choose again. If there are no eligible points, the ants will directly return back to the distribution center of emergency rescue. 


\subsection{Pheromone Update Strategy}

The pheromone update strategy is one of the key steps of ant colony algorithm, information updating too fast will cause the algorithm to trap into local optimum, and even stagnation; if information updates too slow, the convergence speed is slow, unable to search the optimal route. Here, the method combining global information update and partial information update is adopted. The update formula of global information is as follows:

$$
\begin{aligned}
& \tau_{i j}=(1-\rho) \tau_{i j}+\rho \Delta \tau_{i j}^{\text {best }} \quad \rho \in(0,1) \\
& \Delta \tau_{i j}^{\text {best }}=1 / o^{\text {best }}
\end{aligned}
$$

In the expression, $o^{\text {best }}$ is the current global optimal target, $\rho$ is a pheromone volatilization factor.

At the same time, after ant finds a feasible sub-solution, the pheromone in road section $(i, j)$ of feasible sub-solution also undertakes local information update.

$$
\tau_{i j}=(1-\zeta) \tau_{i j}+\zeta \tau_{0} \quad \zeta \in(0,1)
$$

In the expression, ${ }^{\tau_{0}}$ is a constant, ${ }^{\tau_{0}}=1 /\left(n O_{\text {min }}^{3}\right), O^{3}$ in is the optimal target mean value of in the last three times.

\subsection{The Optimization Improvement of 2-opt Method}

In order to further optimize the calculated route, this paper adopts 2-opt method to each ant route produced by each ant [15] to do local improvement, which can eliminate the intersection.

The method is as follows: in the optimum solution of the stage try to replace $(i, i+1)$, $(j, j+1)$ instead of $(i, j),(i+1, j+1)$, if after exchange the decision target value is more optimal and satisfies the constraints, complete the exchange and update the route.

\subsection{Chaos Perturbation Strategy}

Judgment method of ant colony algorithm into the premature and stagnation phenomenon is in the process of ant colony algorithm operation, if the optimal results of each loop calculation do not change after repeating several times, the algorithm is regarded into the status of precocity and stagnation. When judging algorithm enters into the premature, stagnation status, the chaos disturbance mechanism may be started to help search jumping out of local optimal.

$$
y_{i+1}=\mu y_{i}\left(1-y_{i}\right) \quad \mathrm{i}=0,1, \ldots ; \mu \in(3.8,4]
$$

In the expression, ${ }^{\mu}$ is a control variable, when $\mu=4$ and $z_{0} \notin\{0,1 / 4,1 / 2,3 / 4,1\}$, the produced logistic sequence is completely in chaotic state.

After the chaotic system is added into the ant colony system algorithm, expression (10) is written as:

$$
\tau_{i j}=(1-\rho) \tau_{i j}+\rho \Delta \tau_{i j}^{b e s t}+\lambda y_{i j} \quad \rho \in(0,1)
$$


Hereinto, ${ }^{y_{i j}}$ is a chaotic variable, which can be obtained through iteration mapping by expression (13); $\lambda$ is an adjustment coefficient, it may be a constant.

\subsection{The Solving Steps of Chaos Ant Colony System Algorithm}

a) Initialization parameters, maximum iterations number ${ }^{N C_{\max }}$, the ants number m, etc., the number of iterations $\mathrm{NC}=0$.

b) Set the ants starting from distribution center of emergency rescue.

c) Choose the moving targets according to expressions (8) (9), and check the constraints. If the vehicle load exceeds the maximum load, returns back to the emergency rescue distribution center; otherwise, moves to the next rescue point, and add this point into tabu table.

d) Check whether tabu table is full. If not, execute step c); otherwise, execute step e).

e) 2-opt optimization method, calculate the objective function and the optimal solution of this cycle, according to the expression (12) the pheromone on the path with the optimal solution is locally update, and record all the optimal solution of the circulation.

f) If the optimal path obtained in the set continuous Q times of iteration has no obvious change, depending on the expression (13) (14) to the pheromone on the path with optimal solution to do global update and disturbance; otherwise according to expression (10) to do global update to the pheromone on the path with the optimal solution.

g) If it does not reach the largest iterations times, go to step b); otherwise, output the optimal solution, terminate the calculation.

\section{Example Analysis}

Assuming that somewhere there is a natural disaster, an emergency rescue center is ordered to send emergency supplies for the adjacent eight rescue points. The first batch of material supply planning has been determined to send three trucks, the maximum loading capacity of each truck is 100 , the optimizing route makes the emergency rescue distribution is optimal overall. For convenient analysis, in this case the emergency rescue distribution center is marked as the No. 0 node, each rescue point is marked as the rest eight nodes, and the planning supply is shown in table 1.

Table 1. The Emergency Material Supply Plan of each Rescue Point

\begin{tabular}{|c|c|c|c|c|c|c|c|c|}
\hline $\begin{array}{c}\text { Rescue } \\
\text { points }\end{array}$ & 1 & 2 & 3 & 4 & 5 & 6 & 7 & 8 \\
\hline $\begin{array}{c}\text { Supply } \\
\text { number }\end{array}$ & 35 & 32 & 50 & 35 & 35 & 30 & 30 & 38 \\
\hline
\end{tabular}

With the model and algorithm discussed in this article, first of all, the relevant experts make a rapid assessment and give the evaluation value between two nodes (including emergency rescue center and the rescue point). Assuming that safety evaluation value and distance evaluation value are shown in table 2 and 3 , in order to reduce the amount of data processing, in this case the cost estimation value is the $10 \%$ of distance evaluation values, no longer an assessment is made alone. 
Table 2. The Distance Evaluation Values between Emergency Distribution Center and Rescue Points

\begin{tabular}{|c|c|c|c|c|c|c|c|c|c|}
\hline$d_{i j}$ & 0 & 1 & 2 & 3 & 4 & 5 & 6 & 7 & 8 \\
\hline 0 & - & 100 & 50 & 70 & 80 & 100 & 50 & 80 & 95 \\
\hline 1 & 100 & - & 56 & 63 & 92 & 66 & 75 & 69 & 56 \\
\hline 2 & 50 & 56 & - & 87 & 66 & 65 & 58 & 83 & 29 \\
\hline 3 & 70 & 63 & 87 & - & 65 & 45 & 63 & 92 & 88 \\
\hline 4 & 80 & 92 & 66 & 65 & - & 84 & 55 & 56 & 33 \\
\hline 5 & 60 & 66 & 65 & 45 & 84 & - & 49 & 38 & 29 \\
\hline 6 & 50 & 75 & 58 & 63 & 55 & 55 & - & 95 & 29 \\
\hline 7 & 80 & 69 & 83 & 92 & 56 & 56 & 95 & - & 86 \\
\hline 8 & 95 & 56 & 29 & 88 & 33 & 33 & 29 & 86 & - \\
\hline
\end{tabular}

Table 3. The Safety Evaluation Value between Emergency Distribution Center and Rescue Points

\begin{tabular}{|c|c|c|c|c|c|c|c|c|c|}
\hline$s_{i j}$ & 0 & 1 & 2 & 3 & 4 & 5 & 6 & 7 & 8 \\
\hline 0 & - & 5 & 4 & 5 & 5 & 1 & 5 & 3 & 4 \\
\hline 1 & 5 & - & 3 & 5 & 3 & 3 & 5 & 3 & 4 \\
\hline 2 & 4 & 5 & - & 5 & 4 & 3 & 5 & 3 & 2 \\
\hline 3 & 5 & 5 & 2 & - & 5 & 3 & 5 & 3 & 4 \\
\hline 4 & 5 & 5 & 3 & 5 & - & 3 & 5 & 3 & 3 \\
\hline 5 & 3 & 5 & 5 & 5 & 5 & - & 5 & 3 & 4 \\
\hline 6 & 5 & 5 & 4 & 5 & 2 & 3 & - & 3 & 5 \\
\hline 7 & 3 & 5 & 5 & 5 & 5 & 3 & 5 & - & 4 \\
\hline 8 & 4 & 5 & 4 & 5 & 5 & 3 & 5 & 3 & - \\
\hline
\end{tabular}

What needs to explain, according to the previous discussion, the assessment matrix is allowed asymmetric to reflect the different situation of two-way distance.

In the computer with $\mathrm{CPU} 1.8 \mathrm{GHz}$ and memory $1 \mathrm{~GB}$, the solution is obtained through

MATLAB 7.1 programming, the relevant parameters are set as follows: $q_{0}=0.6$, $\alpha=1, \beta=2, \lambda=1, \gamma=1, \rho=0.2, \zeta=0.1, m=50,{ }^{N} C_{\max }=100, Q=5$.

According above data to execute the program (which is marked as case 1), there are three optimized paths, they are $0 \rightarrow 4 \rightarrow 7 \rightarrow 5 \rightarrow 0,0 \rightarrow 6 \rightarrow 8 \rightarrow 2 \rightarrow 0,0 \rightarrow 1 \rightarrow 3 \rightarrow 0$.

The load capacities of the three paths are respectively $100,100,85$, which satisfies the vehicle constraints, hereinto, two trucks are full already. The distance optimal values of three paths are respectively 234,158,233, and the total distance optimal value is 625 .

In order to test the sensitivity of the algorithm, the ${ }^{d_{04}}$ and ${ }^{s_{04}}$ in the optimal route take the worst values, that is, $s_{04}=1, d_{04}=100$, the other values keep invariant( it is denoted as case 2 ). The program runs again to get the optimized the route as $0 \rightarrow 3 \rightarrow 5 \rightarrow 0$, $0 \rightarrow 2 \rightarrow 8 \rightarrow 6 \rightarrow 0,0 \rightarrow 1 \rightarrow 7 \rightarrow 4 \rightarrow 0$, the total distance optimal value is changed into 638 . 
From the optimization result of case 2 , in the line there is no the road section $0 \rightarrow 4$ in the optimization results of case 1, which means the algorithm does not repeat the previous result, but according to the principles of the global optimal to find the alternative route. It is shown that the $4 \rightarrow 0$ road section in the optimal route is obviously superior to the $0 \rightarrow 4$ road section after changing the data, which means the algorithm can effectively identify the directed road section. Therefore, it can be used to the selection of directed road section.

In order to test the performance of the designed algorithm in this paper, the data of first two cases are respectively operated 10 times adopting three kinds of algorithm, which are the designed algorithm in this paper (denoted as chaos disturbance ant colony algorithm), the ordinary ant colony algorithm, and the standard ant colony system (ACS) algorithm, the optimal value can all be obtained, but the performance operation is different, the worst results are shown in table 4 . These can be seen from the simulation results.

Table 4. Compare of Algorithm Results

\begin{tabular}{|c|c|c|c|c|}
\hline Algorithm & performance & Case 1 & Case 2 & Random case \\
\hline \multirow{2}{*}{ General ant colony algorithm } & Iteration times & 10 & 11 & 35 \\
\cline { 2 - 5 } & Optimizing time/s & 1.3 & 1.3 & 17.6 \\
\hline \multirow{2}{*}{ ACS algorithm } & Iteration times & 11 & 11 & 29 \\
\cline { 2 - 5 } & Optimizing time/s & 1.3 & 1.4 & 14.9 \\
\hline $\begin{array}{c}\text { Chaos disturbance } \\
\text { ant colony algorithm }\end{array}$ & Iteration times & 8 & 9 & 26 \\
\cline { 2 - 5 } & Optimizing time/s & 1.1 & 1.3 & 13.3 \\
\hline
\end{tabular}

In order to test the adaptability and stability of this algorithm to deal with the large-scale complex problem, and the data of 30 nodes are taken using random matrix function via three kinds of algorithms to respectively calculate 10 times and take the average value again, its optimum performance contrast situation is shown in the last column of table 4. It is shown from the table, for large-scale problem, the chaotic disturbance improved ant colony algorithm designed in this paper is more apparent comparing with other two kinds of ant colony algorithm in performance difference, which can converge to the optimal solution in faster speed and better stability. Above test shows the design algorithm in this paper can meet the request of more reliable and more efficient of emergency rescue vehicle to scheduling optimization algorithm.

\section{Conclusion}

This paper discusses the decision environment of emergency rescue vehicle scheduling and the actual demands, puts forward the three dimensional optimization indexes and comprehensive evaluation methods to time, cost and safety, based on this foundation multiobjective optimization model of emergency rescue vehicle scheduling is established, and chaos disturbance mechanism is introduced to improve the ant colony system algorithm. At the same time, in the algorithm the links are all optimal designed such as the heuristic factor structure, state transition probability, eliminating intersection point, and pheromone updating etc.. At last, through the experiment comparing, the effectiveness of the algorithm is verified by an experiment comparison. If the model and the algorithm are applied for the decisionmaking of emergency rescue, which can improve the emergency response ability and decision-making efficiency; it is a kind of method to be promoted. 


\section{References}

[1] Chunlin Liu, Jianmin He and Jianjun Shi. A kind of emergency supplies scheduling optimization model research. Journal of management science in China, 9 (3): 26-29(2001).

[2] Zhengwen $\mathrm{He}$, Tao Jiao and $\mathrm{Xu} \mathrm{Yu}$. Based on the prohibited time window of emergency supplies scheduling vehicle routing problem. Journal of operation and management, 19 (2):1-6 (2009).

[3] Ziyao Li. Emergency rescue vehicle routing optimization based on multi-objective improved ant colony algorithm . Journal of technology economy and management research, 9:7-10 (2011).

[4] Wuyang Liu. The logistics optimization and scheduling research in emergency disposal. Journal of Xiangtan university (natural science). 32 (3): 122-126 (2013).

[5] Zhipeng Xu, Jiazhen Peng and Weisheng Xu. Partial delivery planning of emergency logistics and ant colony optimization solution. Computer engineering and application. 47 (24) : 1-3, 8(2011).

[6] Liansheng Tang, Wenming Cheng and Jian Liang. The ant clustering algorithm research of emergency logistics distribution problem. Journal of railway transportation and economy. 30 (9): 66 -69 (2008).

[7] Minghua Chen, Yingqiuli and Yaoqi Luo. The study of emergency logistics vehicle allocation problem. Computer engineering and application, 45(24): 194-197 (2009).

[8] ]Xiaoyong Tan and Qiuju Liu. The research review and prospect of emergency distribution vehicle scheduling optimization . Computer application research, 29 (9): 3212-3215 (2012).

[9] Wei Yi and Arun K. Ant colony optimization for disaster relief operations. Transportation Research Part E. 43(6):660-672(2012).

[10] Lee C. Y., Lee Z. J. and Lin S. W. An enhanced ant colony optimization(EACO)applied to capacitated vehicle routing problem. Applied Intelligence. 32(1):88-95 (2010).

[11] Liyi Zhang, Fei Teng and Ting Liu. The emergency logistics path optimization algorithm based on chaos ant colony algorithm. Journal of China civil aviation university. 29 (3): 61-64(2011).

[12] Hongli Xu, Xu Qian and Yue Xun. A new adaptive chaos ant colony optimization algorithm based on logistic chaotic image to solve the dynamic vehicle routing problem. Computer application research, 29 (6): 20582060 (2012).

[13] Bullheimer B., Hartl R. F., Strauss C. An improved ant system algorithm for the vehicle routing problem. Annals Operations Research. 89(4): 319-328 (2006).

[14] Bell J E and Mcmullen P R. Ant colony optimization techniques for the vehicle routing problem. Advanced Engineering Informatics. 18(1): 41-48 (2004).

[15] Bianchi L, Knowles $\mathbf{J}$ and Bowler J. Local search for the probabilistic traveling salesman problem: correction to the 2-p-opt and 1-shift algorithms. European Journal of Operational Research. 162(1):206-219 (2005).

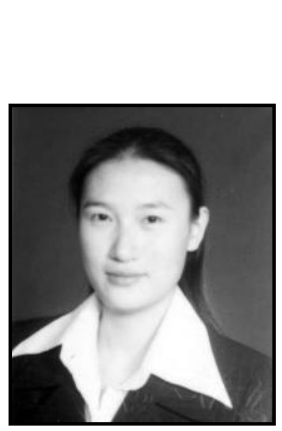

\section{Authors}

Lingxia Liu, she was born in 1976.5, she incepted her master degree in Shanxi University in 2003, and her major is information technology. Now she is working in Computer Education Department of Anyang Normal University as an associate professor. She is also a member of China Computer Federation.

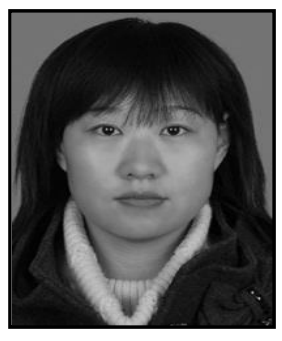

Xuexia Gao, she was born in 1974.11, she got her bachelor degree from the First Air force Aviation College of the Chinese people's liberation army with major of computer engineering. She graduated from Huazhong University of Science Technology with her master degree of software engineering. Now she is a PhD. Candidate in Wuhan University of computer application specialty. She is working in Xinxiang University as an associate professor, and she is a young backbone teacher in Henan Province. 


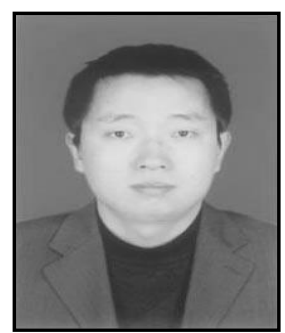

Qiang Song, he was born in 1971.2, he incepted his double master degrees in Ateneo de Manila University in the Philippines in 2005, which are MSIT and MBA. Now he is a PhD. candidate in Wuhan University of Technology in major of information engineering. He is working in Anyang Institute of Technology as an associate professor, and he is also a senior member of China Computer Federation. 
International Journal of Security and Its Applications Vol. 10, No. 2 (2016) 